\title{
Efficient quantum circuits for quantum computational chemistry
}

\author{
Yordan S. Yordanov, ${ }^{1}$ David R. M. Arvidsson-Shukur, ${ }^{1,2}$ and Crispin H. W. Barnes ${ }^{1}$ \\ ${ }^{1}$ Cavendish Laboratory, Department of Physics, University of Cambridge, Cambridge CB3 OHE, United Kingdom \\ ${ }^{2}$ Research Laboratory of Electronics, Massachusetts Institute of Technology, Cambridge, Massachusetts 02139, USA
}

\begin{abstract}
Molecular quantum simulations with the variational quantum eigensolver (VQE) rely on ansatz states that approximate the molecular ground states. These ansatz states are generally defined by parametrized fermionic excitation operators and an initial reference state. Efficient ways to perform fermionic excitations are vital for the realization of the VQE on noisy intermediate-scale quantum computers. Here, we address this issue by first demonstrating circuits that perform qubit excitations, excitations that do not account for fermionic anticommutation relations. We then extend the functionality of these circuits to perform fermionic excitations. Compared to circuits constructed with the standard use of "CNOT staircases", our circuits offer a linear reduction in the number of $C N O T$ gates, by a factor of 2 and 8 per single and double excitation, respectively. Our results reduce the requirements for near-term realizations of quantum molecular simulations.
\end{abstract}

\section{INTRODUCTION}

It is anticipated that quantum computers will be able to simulate quantum systems exponentially faster than classical computers [1-3]. A promising method to perform this task on emerging noisy intermediatescale quantum (NISQ) computers [4, 5] is the variationalquantum-eigensolver (VQE) algorithm [6-9]. The VQE is a hybrid classical-quantum variational algorithm that determines the eigenvalues of a Hamiltonian operator by minimizing its expectation value, with respect to a parametrized ansatz state. The VQE can be used to solve the electronic structure problem, determining the ground-state energy eigenvalue of a molecular Hamiltonian $[10,11]$. Compared to other purely quantum algorithms for eigenvalue-determination, e.g., the quantum phase-estimation algorithm $[12,13]$, the VQE uses shallower quantum circuits and is more error tolerant [6], at the expense of requiring a greater number of measurements and more classical processing.

Two major challenges in the practical realization of the VQE on NISQ computers, are the design of ansatz states, and the construction of efficient circuits to create these states. Most ansatz states, currently considered by the scientific community, correspond to applying a series of fermionic unitary evolutions to an initial reference state, e.g., the Hartree-Fock state. These fermionic unitary evolutions, which we will refer to as "fermionic excitations", are exponentials of parametrized single- and double-fermionic-excitation operators. Examples of such fermionic ansatz states include unitary coupled cluster (UCC) ansatz states [6, 14-16], and states constructed by iterative-VQE algorithms, e.g. the adaptive derivative-assembled pseudo-trotter (ADAPT) VQE $[17,18]$. The standard circuits for fermionic excitations [10, 14, 19, 20] use "CNOT staircases" (see Sec. II A) inefficiently, in terms of CNOT gates. The ability to implement a sufficient number of entangling gates with high enough fidelity is the current bottleneck in NISQ computing [4, 21, 22]. Therefore, more efficient fermionicexcitation circuits will improve the prospects of near- term realization of the VQE on NISQ computers.

In this work, we demonstrate a methodology to construct circuits, optimized in the number of CNOT gates and in the CNOT circuit depth, that perform single and double fermionic excitations. First, we construct simpler excitations that do not account for fermionic anticommutation relations. We call these simplified excitations "qubit excitations". Single qubit excitations can be implemented by an exchange-interaction circuit [2326]. Second, we use this exchange-interaction circuit as a subcircuit to construct a double qubit excitation circuit. Finally, we modify these qubit excitations circuits, to account for fermionic anticommutation relations. In this way we construct circuits that offer a linear reduction in the number of $C N O T$ gates by a factor of 2 and 8 per single and double fermionic excitation, respectively, compared to circuits constructed entirely with $C N O T$ staircases.

The paper is organised as follows: In Sec. II A, we review the standard way of using CNOT staircases to construct fermionic-excitation circuits. In Sec. II B, we outline a method, which is used throughout the paper, to implement multi-qubit-controlled rotations. In Sec. III A, we design circuits that perform single and double qubit excitations. In Sec. IIIB, we utilise our qubit excitations to construct circuits that perform fermionic excitations. We summarize our results in Sec. IV.

\section{THEORY}

Throughout this paper we assume the Jordan-Wigner encoding [10, 27], where the occupancy of the $i^{\text {th }}$ molecular spin orbital is represented by the state of qubit $q_{i}$. We denote spin orbitals with indices $i, j, k, l$, ordered as $i<j<k<l$. 


\section{A. Fermionic excitations}

In this section, we provide a brief description of what a fermionic excitation is, within the context of molecular VQE simulations. We also review the standard method to construct circuits that perform fermionic excitations $[10,14,19,20]$.

We use the term fermionic excitation to refer to an exponential of $\theta$-parametrized fermionic excitation operators. Single and double fermionic excitation operators are defined, respectively, by the skew-Hermitian operators

$$
\begin{gathered}
T_{i}^{k}(\theta) \equiv \theta\left(a_{k}^{\dagger} a_{i}-a_{i}^{\dagger} a_{k}\right) \text { and } \\
T_{i j}^{k l}(\theta) \equiv \theta\left(a_{k}^{\dagger} a_{l}^{\dagger} a_{i} a_{j}-a_{i}^{\dagger} a_{j}^{\dagger} a_{k} a_{l}\right),
\end{gathered}
$$

where $a_{i}^{\dagger}$ and $a_{i}$ denote fermionic creation and annihilation operators, respectively. Single and double fermionic excitations are expressed, respectively, by the unitary operators

$$
\begin{gathered}
U_{k i}^{(\mathrm{sf})}(\theta)=e^{T_{i}^{k}(\theta)} \text { and } \\
U_{k l i j}^{(\mathrm{df})}(\theta)=e^{T_{i j}^{k l}(\theta)} .
\end{gathered}
$$

The operators $a_{i}$ and $a_{i}^{\dagger}$ obey the anticommutation relations

$$
\left\{a_{i}, a_{j}^{\dagger}\right\}=\delta_{i, j} \text { and }\left\{a_{i}, a_{j}\right\}=\left\{a_{i}^{\dagger}, a_{j}^{\dagger}\right\}=0 .
$$

Within the Jordan-Wigner encoding, $a$ and $a^{\dagger}$ can be written in terms of quantum-gate operators as

$$
\begin{gathered}
a_{i}=Q_{i} \prod_{r=0}^{i-1} Z_{r}=\frac{1}{2}\left(X_{i}+i Y_{i}\right) \prod_{r=0}^{i-1} Z_{r} \text { and } \\
a_{i}^{\dagger}=Q_{i}^{\dagger} \prod_{r=0}^{i-1} Z_{r}=\frac{1}{2}\left(X_{i}-i Y_{i}\right) \prod_{r=0}^{i-1} Z_{r}
\end{gathered}
$$

where the qubit creation and annihilation operators are

$$
Q_{i}^{\dagger} \equiv \frac{1}{2}\left(X_{i}-i Y_{i}\right) \text { and } Q_{i} \equiv \frac{1}{2}\left(X_{i}+i Y_{i}\right),
$$

respectively. $Q_{i}$ and $Q_{i}^{\dagger}$ (discussed further in Sec. III A) act to change the occupancy of orbital $i$. The product of Pauli- $z$ operators, $\prod_{r=0}^{i-1} Z_{r}$, acts as an exchange-phase factor, accounting for the anti-commutation relations of $a$ and $a^{\dagger}$.

Using Eqs. (6) and (7), a single fermionic excitation [Eq. (3)] can be re-expressed in terms of quantum-gate operators:

$$
\begin{aligned}
U_{k i}^{(\mathrm{sf})}(\theta)= & \exp \left[-i \frac{\theta}{2}\left(X_{i} Y_{k}-Y_{i} X_{k}\right) \prod_{r=i+1}^{k-1} Z_{r}\right] \\
= & \exp \left[-i \frac{\theta}{2} X_{i} Y_{k} \prod_{r=i+1}^{k-1} Z_{r}\right] \\
& \times \exp \left[i \frac{\theta}{2} Y_{i} X_{k} \prod_{r=i+1}^{k-1} Z_{r}\right] .
\end{aligned}
$$

The exponential $\exp \left[-i \frac{\theta}{2} X_{i} Y_{k} \prod_{r=i+1}^{k-1} Z_{r}\right]$ in Eq. (10), can be implemented by the circuit in Fig. 1. The two CNOT "staircases" together with the $R_{z}(\theta)$ rotation in between them (Fig. 1), are referred to as a "CNOTstaircase construction". This construction implements $U_{\text {stair }} \equiv \exp \left[i \theta \prod_{r=i}^{k} Z_{r}\right]$. The $H$ and $R_{x}\left( \pm \frac{\pi}{2}\right)$ gates, on both sides of the CNOT staircase construction in Fig. 1 act to transform the $Z_{i}$ and the $Z_{k}$ operators, in $U_{\text {stair }}$, to $X_{i}$ or $Y_{k}$ operators, respectively. Similarly, by sandwiching a $C N O T$ staircase construction between single-qubit rotations that transform individual Pauli- $z$ operators to Pauli- $x$ or Pauli- $y$ operators, any exponential of a product of Pauli operators can be constructed. Hence, the single fermionic excitation [Eq. (10)] can be implemented by a circuit that contains $2 C N O T$ staircase constructions. The full circuit for a single fermionic excitation, constructed using the aforementioned method, is included in appendix A Fig. 10.

A double fermionic excitation [Eq. (4)] can be reexpressed in terms of quantum-gate operators:

$$
\begin{array}{r}
U_{k l i j}^{(\mathrm{df})}(\theta)=\exp \left[-i \frac{\theta}{8}\left(X_{i} Y_{j} X_{k} X_{l}+Y_{i} X_{j} X_{k} X_{l}\right.\right. \\
+Y_{i} Y_{j} Y_{k} X_{l}+Y_{i} Y_{j} X_{k} Y_{l}-X_{i} X_{j} Y_{k} X_{l}-X_{i} X_{j} X_{k} Y_{l} \\
\left.\left.-Y_{i} X_{j} Y_{k} Y_{l}-X_{i} Y_{j} Y_{k} Y_{l}\right) \prod_{r=i+1}^{j-1} Z_{r} \prod_{r^{\prime}=k+1}^{l-1} Z_{r^{\prime}}\right] .
\end{array}
$$

$U_{k l i j}^{(\mathrm{df})}(\theta)$ can be implemented by 8 CNOT-staircase constructions. The full circuit for a double fermionic excitation, constructed using the aforementioned method, is included in appendix A Fig. 11.

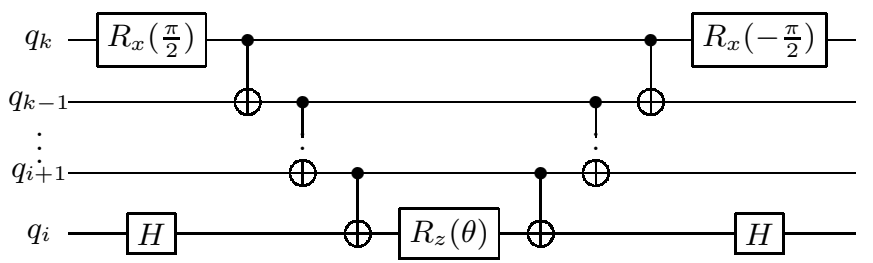

FIG. 1. A circuit implementing the exponential exp [$\left.i \theta X_{i} Y_{k} \prod_{r=i+1}^{k-1} Z_{r}\right]$. 


\section{B. Multi-qubit-controlled rotations}

In this section, we outline a method, used in [28], to construct circuits that implement multi-qubit-controlled rotations. The circuits use no ancilla qubits. Later, we use this method to construct circuits for qubit excitations (see Sec. III A).

We denote a $R_{y}(\theta)$ rotation on a target qubit $q_{0}$, controlled by the $\left\{q_{1} . . q_{m}\right\}$ qubits being in the state $|1 . .1\rangle$, as $R_{y}\left(\theta,\left\{q_{1} . . q_{m}\right\}, q_{0}\right)$. We then write an $m$-qubit-controlled rotation by decomposing it into two opposite half-way rotations, controlled by $(m-1)$ qubits:

$$
\begin{array}{r}
R_{y}\left(\theta,\left\{q_{1} . . q_{m}\right\}, q_{0}\right)= \\
\operatorname{CNOT}\left(q_{1}, q_{0}\right) R_{y}\left(-\frac{\theta}{2},\left\{q_{2} . . q_{m}\right\}, q_{0}\right) \\
\times \operatorname{CNOT}\left(q_{1}, q_{0}\right) R_{y}\left(\frac{\theta}{2},\left\{q_{2} . . q_{m}\right\}, q_{0}\right),
\end{array}
$$

or equivalently, as

$$
\begin{array}{r}
R_{y}\left(\theta,\left\{q_{1} . . q_{m}\right\}, q_{0}\right)= \\
R_{y}\left(\frac{\theta}{2},\left\{q_{2} . . q_{m}\right\}, q_{0}\right) \operatorname{CNOT}\left(q_{1}, q_{0}\right) \\
\times R_{y}\left(-\frac{\theta}{2},\left\{q_{2} . . q_{m}\right\}, q_{0}\right) \operatorname{CNOT}\left(q_{1}, q_{0}\right) .
\end{array}
$$

By decomposing the controlled rotations further, the overall operation is brought down to CNOTs and singlequbit rotations. Implementing directly Eqs. (12) or (13), results in a circuit with $\left(2^{m+1}-2\right) C N O T$ s. However, for $m>2$, Eqs. (12) and (13) can be combined alternately to cancel adjacent CNOTs (see Fig. 2), and obtain a circuit with $\left(2^{m-1}+2\right)$ CNOTs. $R_{z^{-}}$and $R_{x}$-controlled rotations can be obtained by additional single-qubit rotations on the target qubit. Equivalently, $C Z$ (controlled-phase) gates can be used instead of CNOT gates in Eqs. (12) and (13); in some scenarios this can be used to cancel adjacent two-qubit gates (see Sec. III A).

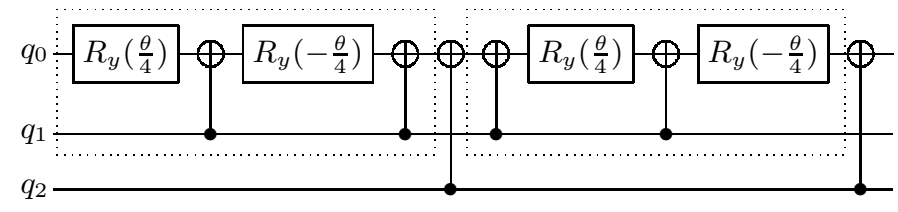

FIG. 2. A circuit implementing $R_{y}\left(\theta,\left\{q_{1}, q_{2}\right\}, q_{0}\right)$. The first half-way rotation $R_{y}\left(\frac{\theta}{2},\left\{q_{1}\right\}, q_{0}\right)$ (left dotted rectangle) is implement as in Eq. (12), and the second half-way rotation $R_{y}\left(-\frac{\theta}{2},\left\{q_{1}\right\}, q_{0}\right)$ ( right dotted rectangle) as in Eq. (13). In this way, the two middle CNOTs between qubits $q_{0}$ and $q_{1}$ can be cancelled.

\section{RESULTS}

In this section we present our main results. We begin by defining qubit excitations. Then, we construct circuits to perform single and double qubit excitations. Last, we modify these circuits to perform single and double fermionic excitations instead.

\section{A. Qubit excitations}

We use the term qubit excitation to describe the unitary evolution of an exponential of a parametrized qubit excitation operator. Single- and double-qubit-excitation operators are generated by the qubit annihilation and creation operators, $Q$ and $Q^{\dagger}$ [Eq. (8)], and are given by the skew-Hermitian operators

$$
\begin{gathered}
\tilde{T}_{i}^{k}(\theta) \equiv \theta\left(Q_{k}^{\dagger} Q_{i}-Q_{i}^{\dagger} Q_{k}\right) \text { and } \\
\tilde{T}_{i j}^{k l}(\theta) \equiv \theta\left(Q_{k}^{\dagger} Q_{l}^{\dagger} Q_{i} Q_{j}-Q_{i}^{\dagger} Q_{j}^{\dagger} Q_{k} Q_{l}\right) .
\end{gathered}
$$

The operators $Q$ and $Q^{\dagger}$ satisfy the relations

$$
\begin{aligned}
& \left\{Q_{i}, Q_{i}^{\dagger}\right\}=I, \quad\left[Q_{i}, Q_{j}^{\dagger}\right]=0 \text { if } i \neq j, \text { and } \\
& {\left[Q_{i}, Q_{j}\right]=\left[Q_{i}^{\dagger}, Q_{j}^{\dagger}\right]=0 \text { for all } i, j .}
\end{aligned}
$$

These relations are neither bosonic nor fermionic; some authors have referred to them as parafermionic [29].

Single and double qubit excitations are expressed, respectively, by the unitaries

$$
\begin{gathered}
U_{k i}^{(\mathrm{sq})}(\theta)=e^{\tilde{T}_{i}^{k}(\theta)} \text { and } \\
U_{k l i j}^{(\mathrm{dq})}(\theta)=e^{\tilde{T}_{i j}^{k l}(\theta)} .
\end{gathered}
$$

These unitary operations are similar to the single and double fermionic excitations [Eqs. (3) and (4)], apart from the absence of the exponentials of products of Pauli$z$ operators that account for the fermionic anticommutation relations [Eq. (5)]. Previously, these operations have been considered in the context of VQE-ansatz construction $[30,31]$.

\section{Circuit for single qubit excitations}

A single qubit excitation [Eq. (17)] can be re-expressed in terms of quantum-gate operators:

$$
U_{k i}^{(\mathrm{sq})}(\theta)=\exp \left[-i \frac{\theta}{2}\left(X_{i} Y_{k}-Y_{i} X_{k}\right)\right]
$$

This unitary is equivalent to an evolution under the exchange interaction, which can be performed by the circuit in Fig. 3. We implement the controlled $R_{y}(\theta)$ rotation in the circuit in Fig. 3a as in Eq. (13), and apply the circuit identity in Fig. 4 to cancel a $C N O T$. In this way we obtain the explicit circuit, for single qubit excitation, shown in Fig. 3b. ${ }^{1}$

\footnotetext{
1 The circuit in Fig. 3b is not optimal in the number of CNOT gates. A single qubit excitation, as defined in Eq. (19), can be implemented by a circuit with only two CNOTs. We use the circuit in Fig. $3 \mathrm{~b}$ because it is used to construct the circuits in Figs. 5, 8 and 9 in an optimal way.
} 
a)

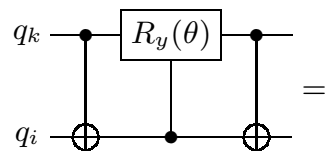

b) $q$

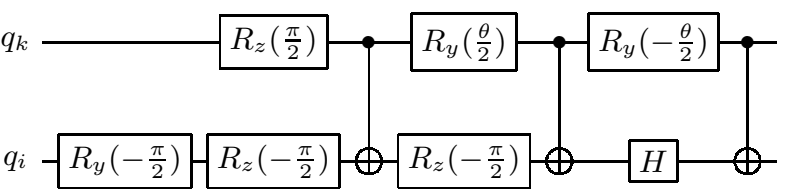

FIG. 3. a) A circuit performing an exchange-interaction operation equivalent to the single qubit excitation in Eq. (19). b) An explicit circuit for a) obtained by implementing the controlled- $R_{y}(\theta)$ rotation as in Eq. (13), and using the circuit identity in Fig. 4.

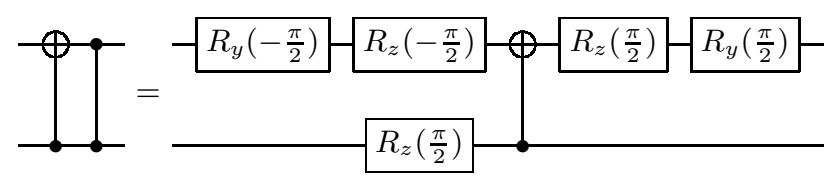

FIG. 4. A circuit identity for an operation equivalent to a $C N O T$ followed by a $C Z$.

\section{Circuit for double qubit excitations}

A double qubit excitation [Eq. (20)] can be reexpressed in terms of quantum-gate operators:

$$
\begin{aligned}
& U_{k l i j}^{(\mathrm{dq})}(\theta)=\exp \left[-i \frac{\theta}{8}\left(X_{i} Y_{j} X_{k} X_{l}+Y_{i} X_{j} X_{k} X_{l}\right.\right. \\
& +Y_{i} Y_{j} Y_{k} X_{l}+Y_{i} Y_{j} X_{k} Y_{l}-X_{i} X_{j} Y_{k} X_{l}-X_{i} X_{j} X_{k} Y_{l} \\
& \left.\left.-Y_{i} X_{j} Y_{k} Y_{l}-X_{i} Y_{j} Y_{k} Y_{l}\right)\right] .
\end{aligned}
$$

Equation (20) is a unitary operation that continuously exchanges the $\left|1_{i} 1_{j} 0_{k} 0_{l}\right\rangle$ and $\left|0_{i} 0_{j} 1_{k} 1_{l}\right\rangle$ states, but does not alter other states. To implement this operation, we use a similar circuit to the one for the single qubit excitation. However, to ensure that the operation exchanges only the states $\left|1_{i} 1_{j} 0_{k} 0_{l}\right\rangle$ and $\left|0_{i} 0_{j} 1_{k} 1_{l}\right\rangle$, it must act nontrivially only if the parities of the qubit pairs $\left\{q_{k} q_{l}\right\}$ and $\left\{q_{i} q_{j}\right\}$ are even. To perform this kind of parity-controlled exchange operation, we use the circuit in Fig. 5. The first two $C N O T \mathrm{~s}$, between qubits $q_{k}$ and $q_{l}$, and qubits $q_{i}$ and $q_{j}$, encode the parities of the two respective qubit pairs on qubits $q_{k}$ and $q_{i}$, respectively. Then qubits $q_{k}$ and $q_{i}$ are used as control qubits for a controlled exchange operation, the dotted rectangle in Fig. 5, between qubits $q_{l}$ and $q_{j}$. The last two CNOTs between qubits $q_{k}$ and $q_{l}$, and qubits $q_{i}$ and $q_{j}$, respectively, reverse the parityencoding action of the first two CNOTs.

We implement the controlled $R_{y}(\theta)$ rotation in Fig. 5 with the circuit in Fig. 6, and use the circuit identity in Fig. 4 to cancel a $C N O T$. In this way, we obtain the explicit circuit for a double qubit excitation, shown in Fig. 7. The explicit circuit has a CNOT count of 13 and CNOT depth of 11 . The circuit suggested in Refs. [31, 32], for the same operation, also has a CNOT count of 13 , but a higher CNOT depth of $13^{2}$.

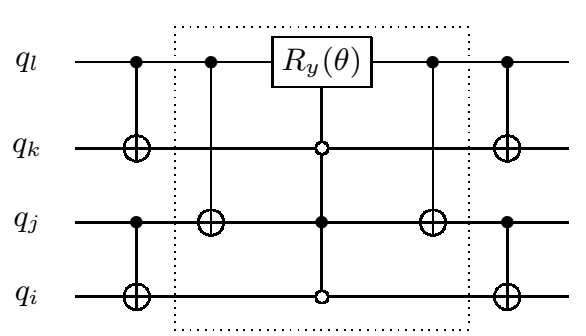

FIG. 5. A circuit performing a double qubit excitation [Eq. (20)]. The explicit circuit is given in Fig. 7.

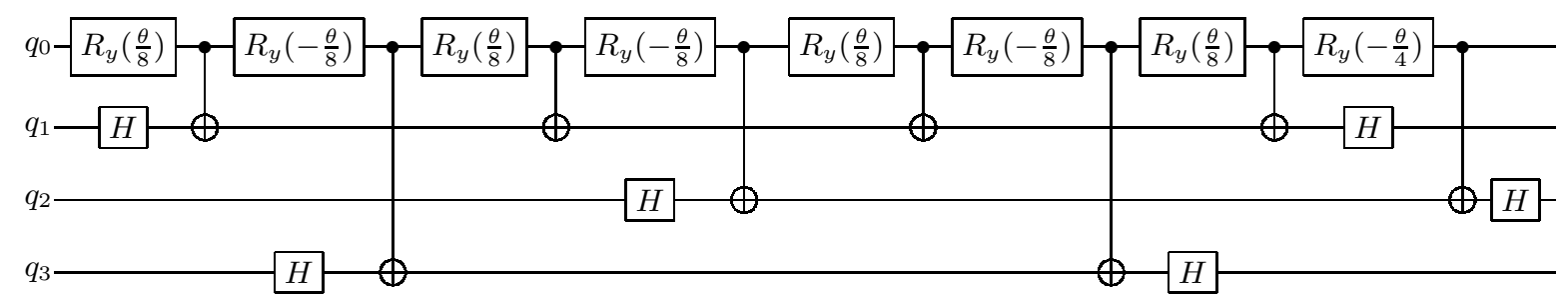

FIG. 6. An explicit circuit implementing the controlled rotation $R_{y}\left(\theta,\left\{q_{1}, q_{2}, q_{3}\right\}, q_{0}\right)$. The circuit is obtained with the method described in Sec. IIB.

\footnotetext{
2 An anonymous referee has pointed out that another advantage of the circuit on Fig. 7 is that it reduces the number of long-range CNOTs compared to the circuits in Refs. [31, 32]
} 


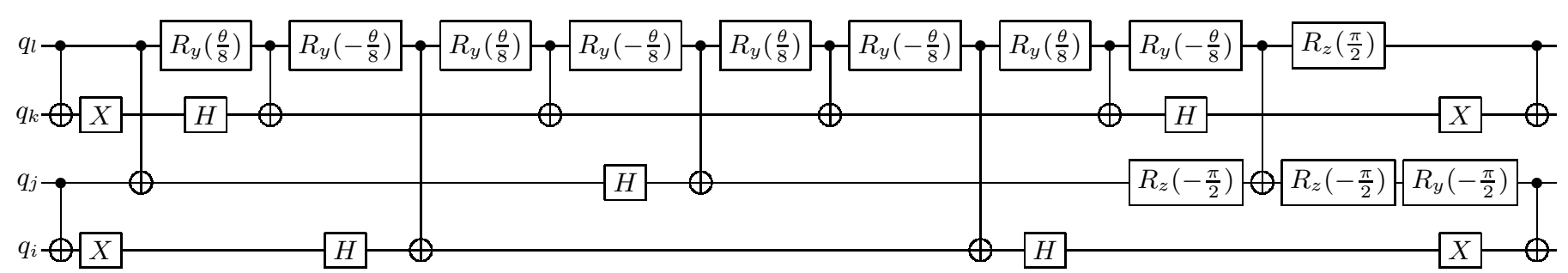

FIG. 7. An explicit circuit implementing a double qubit excitation [Eq. (20)].

\section{B. Efficient Fermionic excitations}

The quantum-gate operator expressions for fermionic excitations [Eqs. (10) and (11)] differ from those for qubit excitations [Eqs. (19) and (20)] only by the additional products of Pauli- $z$ operators in their exponents. These products account for the fermionic anticommutation relations [Eq. (5)]: In the single (double) fermionic excitation, the operator product changes the sign before the excitation parameter $\theta$ if the parity of the set of qubits $\left\{q_{i+1} . . q_{k-1}\right\}\left(\left\{q_{i+1} . . q_{j-1} q_{k+1} . . q_{l-1}\right\}\right)$ is odd. We can reexpress the fermionic excitations in terms of qubit excitations:

$$
\begin{aligned}
& U_{k i}^{(\mathrm{sf})}(\theta)=\left\{\begin{array}{l}
U_{k i}^{(\mathrm{sq})}(\theta) \text { if } P\left(q_{i+1} . . q_{k-1}\right)=0 \\
U_{k i}^{(\mathrm{sq})}(-\theta) \text { if } P\left(q_{i+1} . . q_{k-1}\right)=1
\end{array}, \quad(21)\right. \\
& U_{k l i j}^{(\mathrm{df})}(\theta)=\left\{\begin{array}{l}
U_{k l i j}^{(\mathrm{dq})}(\theta) \text { if } P\left(q_{i+1} . . q_{j-1} q_{k+1} . . q_{l-1}\right)=0 \\
U_{k l i j}^{(\mathrm{dq})}(-\theta) \text { if } P\left(q_{i+1} . . q_{j-1} q_{k+1} . . q_{l-1}\right)=1
\end{array}\right.
\end{aligned}
$$

We can adapt the circuits for qubit excitations in Figs. 3 and 5 to perform fermionic excitation, by sandwiching the controlled- $R_{y}(\theta)$ rotation in each of them between two CNOT staircases [see Figs. 8 and 9]. In this way the sign before the excitation parameter $\theta$ is changed if the parity of the relevant qubits is odd, in accordance with Eqs. (21) and (22). Compared to the standard circuits for fermionic excitations, (see appendix A), the circuits outlined here utilizes only 2 CNOT staircases, instead of 4 or 16, per single or double fermionic excitation, respectively.

\section{Circuit for single fermionic excitations}

Figure 8 shows our modified circuit for a single fermionic excitation, based on the circuit in Fig. 3a. The parity of $\left\{q_{i+1} . . q_{k-1}\right\}$ is encoded in qubit $q_{i+1}$ by a CNOT staircase. Conditioned on qubit $q_{i-1}$ being in state $|1\rangle$ (odd parity), the two $C Z$ gates between qubits $q_{k}$ and $q_{i+1}$ reverse the direction of the $R_{y}(\theta)$ rotation:
$R_{y}(\theta) \rightarrow R_{y}(-\theta)$. The controlled $R_{y}(\theta)$ rotation is implemented as in the single-qubit-excitation circuit, and similarly the circuit identity from Fig. 4 is used to cancel a CNOT between qubits $q_{k}$ and $q_{i}$. A single fermionic excitation, Eq. (10), involves operations on qubits $q_{i}$ to $q_{k}$. We define the total number of qubits involved in the excitation as $n^{(\mathrm{sf})} \equiv k-i+1$. For $n^{(\mathrm{sf})} \geq 3$, the circuit in Fig. 8 has a CNOT count of $2 n^{(\mathrm{sf})}-1$ and a $C N O T$ depth of $\max \left[5,2 n^{(\mathrm{sf})}-3\right]$. For $n^{(\mathrm{sf})}=2$, the circuit in Fig. 8 is equivalent to that for a single qubit excitation (Fig. 3b). For comparison, the standard construction (Sec. II A) for single-fermionic-excitation circuits, yields a circuit (appendix A Fig. 10) with a CNOT count and a CNOT depth of $4\left(n^{(\mathrm{sf})}-1\right)$.

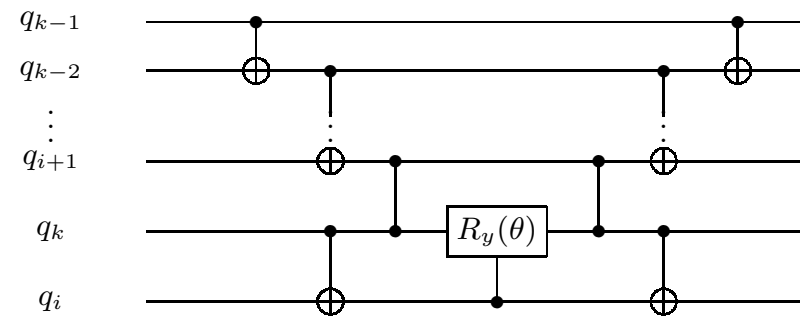

FIG. 8. A circuit performing a single fermionic excitation [Eq. (10)]. The vertical dots denote CNOT staircases on qubits $q_{i+1}$ to $q_{k-1}$.

\section{Circuit for double fermionic excitations}

Figure 9 shows our modified circuit for a double fermionic excitation, based on the circuit in Fig. 5. The parity of $\left\{q_{i+1} . . q_{j-1} q_{k+1} . . q_{l-1}\right\}$ is encoded in qubit $q_{i+1}$ by a staircase CNOT structure. Conditioned on qubit $q_{i+1}$ being in state $|1\rangle$, the two $C Z$ gates between qubits $q_{l}$ and $q_{i+1}$ reverse the direction of the controlled $R_{y}(\theta)$ rotation: $R_{y}(\theta) \rightarrow R_{y}(-\theta)$. The controlled $R_{y}(\theta)$ rotation is implemented as in Fig. 6, and the circuit identity from Fig. 4 is used to cancel a CNOT between qubits $q_{l}$ and $q_{j}$. A double fermionic excitations, Eq. (11), involves operations on qubits $q_{i}$ to $q_{j}$ and $q_{k}$ to $q_{l}$. We define the total number of qubits participating in the excitation as $n^{(\mathrm{df})} \equiv j-i+l-k+2$. The circuit in Fig. 9 has a CNOT count of $2 n^{(\mathrm{df})}+5$ and a CNOT depth of $\max \left[13,2 n^{(\mathrm{df})}-1\right]$ for $n^{(\mathrm{df})} \geq 5$. For $n^{(\mathrm{df})}=4$, the circuit in Fig. 9 is equivalent to that for a double qubit excitation (Figs. 5 and 7). For comparison, the standard 


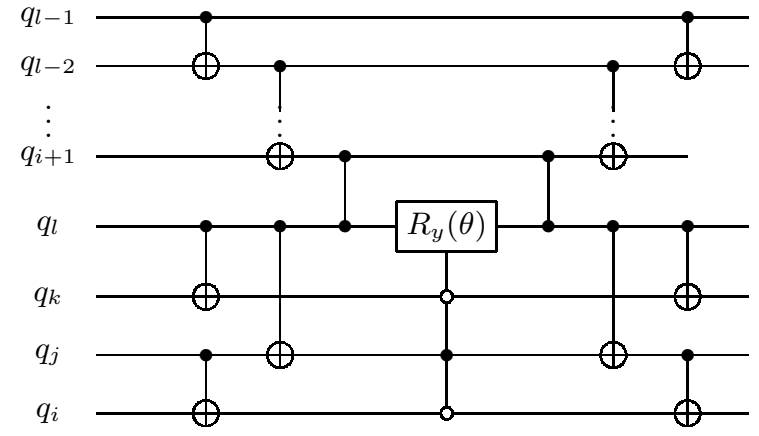

FIG. 9. A circuit performing a double fermionic excitation [Eq.(11)]. The vertical dots denote CNOT staircases on qubits $q_{i+1}$ to $q_{j-1}$, and $q_{k+1}$ to $q_{l-1}$.

construction (Sec. II A) for fermionic-excitation circuits, yields a circuit (appendix A Fig. 11) with a CNOT count and a $C N O T$ depth of $16\left(n^{(\mathrm{df})}-1\right)$. Additionaly, using the method of "balanced trees", suggested in Ref. [33], one can rearrange the CNOTs in the fermionic excitation circuits (Figs. 8 and 9), so that their CNOT depths grow logarithmically rather than linearly.

\section{SUMMARY}

In this work, we constructed circuits, optimized in terms of CNOT gates, to perform efficiently single and double qubit excitations, excitations generated by qubit creation and annihilation operators. We then expanded the circuits' functionality to account for fermionic anticommutation relations, and to perform fermionic excitations. Our single-fermionic-excitation circuits use $2 n^{(\mathrm{sf})}-1$ CNOTs, and have CNOT depths of 3 for $n^{(\mathrm{sf})}=2$, and $\max \left[5,2 n^{(\mathrm{sf})}-3\right]$ for $n^{(\mathrm{sf})} \geq 3$, where $n^{(\mathrm{sf})}$ is the number of orbitals on which the single fermionic excitation acts. Our double-fermionic-excitation circuits use $2 n^{(\mathrm{df})}+5$ CNOTs, and have CNOT depths of 11 for $n^{(\mathrm{df})}=4$, and $\max \left[13,2 n^{(\mathrm{df})}-1\right]$ for $n^{(\mathrm{df})} \geq 5$, where $n^{(\mathrm{df})}$ is the number of orbitals on which the double fermionic excitation acts. To our knowledge, our circuits are more efficient than all previous methods for implementing double qubit and double fermionic excitationsboth in CNOT count and in CNOT depth. Our optimized circuits present a significant step towards implementations of VQE algorithms on NISQ devices.

\section{ACKNOWLEDGMENTS}

The authors wish to thank V. Armaos for useful discussions, and an anonymous referee for pointing out the advantage of our methods mentioned in Footnote 2. Y.S.Y. acknowledges financial support from the EPSRC and Hitachi via CASE studentship RG97399. D.R.M.A.-S. was supported by the EPSRC, Lars Hierta's Memorial Foundation and Girton College.

\section{Appendix A: Standard single and double fermionic excitations circuits}

In this appendix, we present the full circuits for single and double fermionic excitations constructed with the standard use of CNOT staircases. As described in Sec. II A, a single fermionic excitation [Eq. (10)] can be implemented by the circuit in Fig. 10, which contains 2 CNOT staircase constructions. Similarly, a double fermionic excitations [Eq. (11)] can be implemented by the circuit in Fig. 11, which contains 8 CNOT staircase constructions.

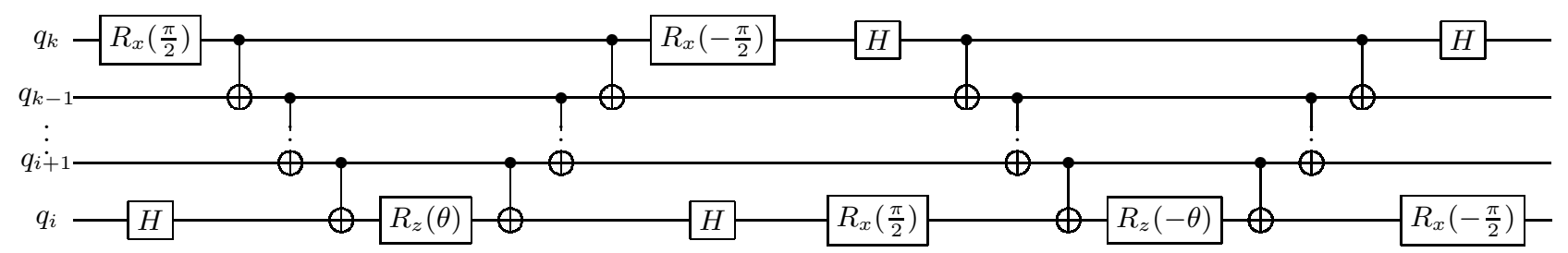

FIG. 10. A standard circuit performing a single fermionic excitation [Eq. (10)]. The vertical dots denote CNOT staircases on qubits $q_{i+1}$ to $q_{k-1}$. 

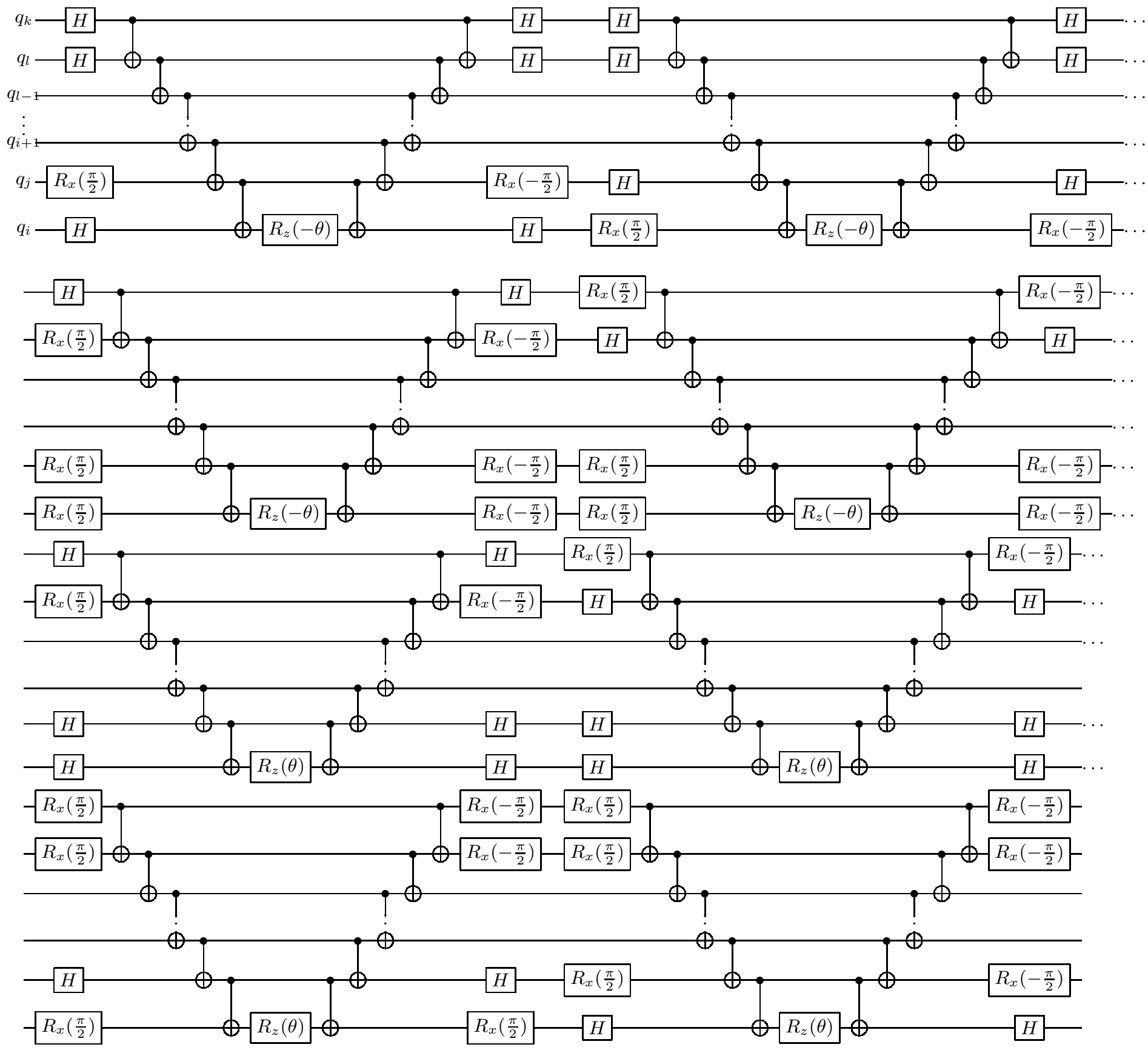

FIG. 11. A standard circuit performing a double fermionic excitation [Eq. (11)]. The vertical dots denote $C N O T$ staircases on qubits $q_{i+1}$ to $q_{j-1}$, and $q_{k+1}$ to $q_{l-1}$.

[1] P. Benioff, Journal of statistical physics 22, 563 (1980).

[2] R. P. Feynman, Int. J. Theor. Phys 21 (1999).

[3] S. Lloyd, Science, 1073 (1996).

[4] J. Preskill, Quantum 2, 79 (2018).

[5] F. Arute, K. Arya, R. Babbush, D. Bacon, J. C. Bardin, R. Barends, R. Biswas, S. Boixo, F. G. Brandao, D. A. Buell, et al., Nature 574, 505 (2019).
[6] A. Peruzzo, J. McClean, P. Shadbolt, M.-H. Yung, X.-Q. Zhou, P. J. Love, A. Aspuru-Guzik, and J. L. O'brien, Nature communications 5, 4213 (2014).

[7] P. J. J. O’Malley, R. Babbush, I. D. Kivlichan, J. Romero, J. R. McClean, R. Barends, J. Kelly, P. Roushan, A. Tranter, N. Ding, B. Campbell, Y. Chen, Z. Chen, B. Chiaro, A. Dunsworth, A. G. Fowler, E. Jeffrey, E. Lucero, A. Megrant, J. Y. Mutus, M. Nee- 
ley, C. Neill, C. Quintana, D. Sank, A. Vainsencher, J. Wenner, T. C. White, P. V. Coveney, P. J. Love, H. Neven, A. Aspuru-Guzik, and J. M. Martinis, Phys. Rev. X 6, 031007 (2016).

[8] D. Wang, O. Higgott, and S. Brierley, Phys. Rev. Lett. 122, 140504 (2019).

[9] F. Arute, K. Arya, R. Babbush, D. Bacon, J. C. Bardin, R. Barends, S. Boixo, M. Broughton, B. B. Buckley, D. A. Buell, et al., arXiv preprint arXiv:2004.04174 (2020).

[10] S. McArdle, S. Endo, A. Aspuru-Guzik, S. C. Benjamin, and X. Yuan, Rev. Mod. Phys. 92, 015003 (2020).

[11] T. Helgaker, P. Jorgensen, and J. Olsen, Molecular electronic-structure theory (John Wiley \& Sons, 2014).

[12] M. A. Nielsen and I. Chuang, "Quantum computation and quantum information," (2002).

[13] U. Dorner, R. Demkowicz-Dobrzanski, B. J. Smith, J. S. Lundeen, W. Wasilewski, K. Banaszek, and I. A. Walmsley, Phys. Rev. Lett. 102, 040403 (2009).

[14] C. Hempel, C. Maier, J. Romero, J. McClean, T. Monz, H. Shen, P. Jurcevic, B. P. Lanyon, P. Love, R. Babbush, A. Aspuru-Guzik, R. Blatt, and C. F. Roos, Phys. Rev. X 8, 031022 (2018).

[15] J. Romero, R. Babbush, J. R. McClean, C. Hempel, P. J. Love, and A. Aspuru-Guzik, Quantum Science and Technology 4, 014008 (2018).

[16] J. Lee, W. J. Huggins, M. HeadGordon, and K. B. Whaley, Journal of chemical theory and computation 15, 311 (2018).

[17] H. R. Grimsley, S. E. Economou, E. Barnes, and N. J. Mayhall, Nature communications 10, 1 (2019).

[18] A. J. M. Daniel Claudino, Jerimiah Wright and T. S. Humble, arXiv preprint arXiv:2011.01279 (2020).

[19] J. D. Whitfield, J. Biamonte, and A. AspuruGuzik, Molecular Physics 109, 735 (2011), https://doi.org/10.1080/00268976.2011.552441.

[20] G. Salis and N. Moll, arXiv preprint arXiv:1908.09533 (2019).

[21] D. M. Zajac, A. J. Sigillito, M. Russ, F. Borjans, J. M. Taylor, G. Burkard, and J. R. Petta, Science 359, 439 (2018).

[22] F. A. Calderon-Vargas, G. S. Barron, X.-H. Deng, A. J. Sigillito, E. Barnes, and S. E. Economou, Phys. Rev. B 100, 035304 (2019).

[23] P. K. Barkoutsos, J. F. Gonthier, I. Sokolov, N. Moll, G. Salis, A. Fuhrer, M. Ganzhorn, D. J. Egger, M. Troyer, A. Mezzacapo, S. Filipp, and I. Tavernelli, Phys. Rev. A 98, 022322 (2018).

[24] M. Ganzhorn, D. Egger, P. Barkoutsos, P. Ollitrault, G. Salis, N. Moll, M. Roth, A. Fuhrer, P. Mueller, S. Woerner, I. Tavernelli, and S. Filipp, Phys. Rev. Applied 11, 044092 (2019).

[25] H. V. Lepage, A. A. Lasek, D. R. M. Arvidsson-Shukur, and C. H. W. Barnes, Phys. Rev. A 101, 022329 (2020).

[26] B. T. Gard, L. Zhu, G. S. Barron, N. J. Mayhall, S. E. Economou, and E. Barnes, npj Quantum Information 6, 1 (2020).

[27] A. Aspuru-Guzik, A. D. Dutoi, P. J. Love, and M. HeadGordon, Science 309, 1704 (2005).

[28] Y. S. Yordanov and C. H. W. Barnes, Phys. Rev. A 100, 062317 (2019).

[29] L.-A. Wu and D. Lidar, Journal of Mathematical Physics 43, 4506 (2002).

[30] R. Xia and S. Kais, Quantum Science and Technology (2020).

31] Y. Nam, J.-S. Chen, N. C. Pisenti, K. Wright, C. Delaney, D. Maslov, K. R. Brown, S. Allen, J. M. Amini, J. Apisdorf, et al., npj Quantum Information 6, 1 (2020).

[32] Q. Wang, M. Li, C. Monroe, and Y. Nam, arXiv preprint arXiv:2004.04151 (2020).

[33] A. Cowtan, S. Dilkes, R. Duncan, W. Simmons, and S. Sivarajah, arXiv preprint arXiv:1906.01734 (2019), 10.4204/EPTCS.318.13. 